\title{
Limiting Behavior of MHD Flow with Hall Current, Due to a Porous Stretching Sheet
}

\author{
Faiza M. N. El-Fayez \\ Department of Mathematics, Princess Nora Bint Abdul Rahman University, Riyadh, KSA \\ Email: fmalfaiz@pnu.edu.sa, dr.faiza5@hotmail.com
}

Received December 2013

\begin{abstract}
An electrically conducting fluid is driven by a stretching sheet, in the presence of a magnetic field that is strong enough to produce significant Hall current. The sheet is porous, allowing mass transfer through suction or injection. The limiting behavior of the flow is studied, as the magnetic field strength grows indefinitely. The flow variables are properly scaled, and uniformly valid asymptotic expansions of the velocity components are obtained through parameter straining. The leading order approximations show sinusoidal behavior that is decaying exponentially, as we move away from the surface. The two-term expansions of the surface shear stress components, as well as the far field inflow speed, compare well with the corresponding finite difference solutions; even at moderate magnetic fields.
\end{abstract}

\section{Keywords}

\section{MHD Flow, Hall Current, Stretching Sheet, Porous Sheet, Limiting Behavior}

\section{Introduction}

The flow due to a moving surface is an important field of fluid mechanics. It has several applications; for example, in production of glass and paper sheets, drawing of plastic films, and extrusion of metals and polymers. Crane [1] obtained an exact solution for the two-dimensional flow of a Newtonian fluid due to a linearly stretching sheet. The velocity components in this case has simple forms; tending to their limits monotonically in an exponential manner as we move away from the surface. The simplicity of this solution invited several authors to consider different related problems, which exhibit the same behavior; e.g. [2]-[8].

One such problem is when the fluid is electrically conducting and a magnetic field is applied normally to the surface. For weak magnetic fields, the flow remains two-dimensional and a closed-form solution is still possible to attain. The Lorentz force acts to restrain the flow; causing faster exponential tendency to the limits. When the magnetic field is strong enough to produce significant Hall current, the problem changes considerably. It becomes three-dimensional. The Hall current is associated with an electromagnetic force which derives a transverse flow. A closed-form solution is no longer possible.

It is interesting to explore the nature of this MHD flow in the presence of Hall current. To that end, the limiting behavior of the flow as the magnetic field grows indefinitely is studied. The straightforward perturbation analysis leads to secular behavior, which is removed by parameter straining [9]. Three-term uniformly valid asy- 
mptotic expansions are, thus, obtained. The presence of the Hall current leads to an exponential tendency to the limits of sinusoidal nature. The flow involves alternating regions of forward and backward velocity components.

Finite difference solutions are also obtained and show qualitative adherence to the predicted limiting behavior even for moderate magnetic fields. Quantitatively, the two term expansions show excellent agreement with the numerical results.

\section{Formulation of the Problem}

A totally ionized fluid is driven by an insulated sheet, which is symmetrically stretching with speed $u_{\mathrm{s}}$ that is proportional to the distance $x$ from the axis of symmetry z. Specifically, $u_{\mathrm{s}}=\omega x$, where $\omega$ is a constant of proportionality. The sheet is porous; allowing a uniform fluid injection of speed $w_{\mathrm{s}}$ in the $\mathrm{z}$ direction. Otherwise, the fluid would have been quiescent. A uniform magnetic field is applied in the z-direction. The magnetic Reynolds number is small, so that the induced magnetic field can be neglected; and the applied field maintains its uniform magnetic flux density $B$. On the other hand, the magnetic field is strong enough to produce significant curvature in the electrons trajectories; leading to considerable Hall current [10].

The fluid is incompressible of density $\rho$, viscosity $\mu$, electrical conductivity $\sigma$, Hall coefficient $h\left(=1 / e n_{\mathrm{e}}\right)$; where $n_{\mathrm{e}}$ is the number density for the electrons and $-e$ is the electron charge. All these parameters are considered constant.

The velocity components: $u$ in the $x$-direction, $v$ in the transverse $y$-direction, and $w$ in the $z$-direction, as well as the pressure $p$ are dependent on $x$ and $z$ only. They are governed by the following continuity and NavierStokes equations, which include components of the Lorentz force corrected for the Hall current,

$$
\begin{gathered}
u_{x}+w_{z}=0 \\
\rho\left(u u_{x}+w u_{z}\right)=\mu\left[u_{x x}+u_{z z}\right]-p_{x}-\frac{\sigma B^{2}}{1+m^{2}}(u-m v) \\
\rho\left(u v_{x}+w v_{z}\right)=\mu\left[v_{x x}+v_{z z}\right]-\frac{\sigma B^{2}}{1+m^{2}}(v+m u) \\
\rho\left(u w_{x}+w w_{z}\right)=\mu\left[w_{x x}+w_{z z}\right]-p_{z}
\end{gathered}
$$

where subscripts denote differentiation, $m=\sigma h B$ is the Hall parameter. Note that $m$ may be positive or negative in accordance with the sign of $B$; i.e. depending on whether the magnetic field is directed away from or toward the sheet. However, as a simultaneous change of the signs of $m$ and $v$ leaves the problem unaltered, only non-negative values of $m$ need to be considered.

At the surface, $z=0$, the adherence conditions $u=\omega x$ and $v=0$ apply; together with the injection condition $w$ $=w_{\mathrm{s}}$. Far from the sheet, as $z \sim \infty$, the fluid has pressure $p_{\infty}$ and velocity components $u \sim 0$ and $\nu \sim 0$.

The problem admits the similarity transformations $z=(\mu / \rho \omega)^{1 / 2} \zeta, u=\omega \times F(\zeta), v=\omega x G(\zeta), w=(\omega \mu / \rho)^{1 / 2} H(\zeta)$, and $p=p_{\infty}+\omega \mu Q(\zeta)$; leading to the following problem.

$$
\begin{gathered}
H^{\prime}+F=0 \\
F^{\prime \prime}-H F^{\prime}-F^{2}-\hat{\beta}(\hat{n} F-\hat{m} G)=0 \\
G^{\prime \prime}-H G^{\prime}-F G-\hat{\beta}(\hat{n} G+\hat{m} F)=0 \\
Q^{\prime}=H^{\prime \prime}-H H^{\prime} \\
\zeta=0: F=1, G=0, H=H_{s} \\
\zeta \sim \infty: F \sim 0, G \sim 0, Q \sim 0
\end{gathered}
$$

where $(\hat{\beta}, \hat{m}, \hat{n})=\frac{(\beta, m, 1)}{\sqrt{1+m^{2}}}$, with $\beta=\frac{\sigma B^{2}}{\rho \omega}$ being the magnetic interaction number; and a dash denotes differrentiation with respect to $\zeta$. 


\section{Asymptotic Analysis}

We are interested in the limiting behavior of the flow as $\beta \sim \infty$ with fixed $m$; i.e. as $\hat{\beta} \sim \infty$ with fixed $\hat{m}$ and $\hat{n}$. That $F(0)=1$ irrespective of the value of $\hat{\beta}$ means that $F=\Theta\left(\hat{\beta}^{0}\right)$. The leading term in Equation (2b) is $\hat{\beta}$ $\hat{n} F$. It can be balanced by the diffusion term $F^{\prime \prime}$ in a contracting region in which $z=\mathcal{O}\left(\hat{\beta}^{-1 / 2}\right)$. Then, Equation (2a) gives $H=\mathcal{O}\left(\hat{\beta}^{-1 / 2}\right)$; consistent with which $H_{\mathrm{s}}$ must be $\Theta\left(\hat{\beta}^{-1 / 2}\right)$. Consequently, Equation (2d) gives $Q=\mathcal{O}\left(\hat{\beta}^{0}\right)$. In Equation (2c), the driving force for the transverse flow is the Hall effect expressed by $\hat{\beta} \hat{m} F$. This requires $G^{\prime \prime} \sim \hat{\beta} \hat{m} F$; leading to $G=\mathcal{O}\left(\hat{\beta}^{0}\right)$.

New stretched variables

$$
\eta=\hat{\beta}^{1 / 2} \zeta, V=\hat{\beta}^{1 / 2} H
$$

are introduced; transforming Problem (2) to the form

$$
\begin{gathered}
V^{\prime}+F=0 \\
F^{\prime \prime}-\hat{n} F+\hat{m} G=\hat{\beta}^{-1}\left(V F^{\prime}+F^{2}\right) \\
G^{\prime \prime}-\hat{n} G-\hat{m} F=\hat{\beta}^{-1}\left(V G^{\prime}+F G\right) \\
Q^{\prime}=V^{\prime \prime}-\hat{\beta}^{-1} V V^{\prime} \\
F(0)=1, G(0)=0, V(0)=V_{s} \\
F(\infty) \sim 0, G(\infty) \sim 0, Q(\infty) \sim 0
\end{gathered}
$$

where, now, the dashes denote differentiation with respect to $\eta$.

We expand the flow variables in powers of $\hat{\beta}^{-1}$ in the form

$$
Z \sim Z_{0}+\hat{\beta}^{-1} Z_{1}+\hat{\beta}^{-2} Z_{2}+\cdots
$$

where $Z$ stands for $F, G, V$, and $Q$. The problems for $Z_{\mathrm{n}}, \mathrm{n}=0,1,2,3, \cdots$, are lin.ear. For $Z_{0}$, we get the basic flow solutions $F_{0}=E C, G_{0}=-E S, V_{0}=V_{\mathrm{s}}+[-\alpha+E(\alpha C-\gamma S)] /\left(\alpha^{2}+\gamma^{2}\right)$, and $Q_{0}=-E C$, where $\alpha$ and $\gamma$ satisfy $\alpha^{2}-\gamma^{2}=\hat{n}$ and $2 \alpha \gamma=\hat{m}$; with $S=\sin \gamma \eta, C=\cos \gamma \eta$ and $E=\exp (-\alpha \eta)$, for short. For $Z_{1}$, the solutions involve secular terms of the form $\eta E S$ and $\eta E C$, the removal of which is effected by straining the parameters $\hat{m}$ and $\hat{n}$ in the form (5); and the procedure can be continued to higher orders.

The following expansions up to $\mathcal{O}\left(\hat{\beta}^{-2}\right)$ are obtained

$$
\begin{gathered}
\hat{n} \sim\left(\alpha^{2}-\gamma^{2}\right)+\hat{\beta}^{-1} \alpha v_{0}+\hat{\beta}^{-2} \alpha v_{1}+\cdots \\
\hat{m} \sim 2 \alpha \gamma+\hat{\beta}^{-1} \gamma v_{0}+\hat{\beta}^{-2} \gamma v_{1}+\cdots \\
F \sim E C+\hat{\beta}^{-1}\left[E\left(C f_{1}+S g_{1}\right)-E^{2} f_{1}\right]+\hat{\beta}^{-2}\left[E\left(C f_{2}+S g_{2}\right)+E^{2} \bar{c}+E^{3}(C \bar{a}+S \bar{b})\right]+\cdots \\
G \sim-E S+\hat{\beta}^{-1}\left[E\left(C g_{1}-S f_{1}\right)-E^{2} g_{1}\right]+\hat{\beta}^{-2}\left[E\left(C g_{2}-S f_{2}\right)+E^{2} \tilde{c}+E^{3}(C \tilde{a}+S \tilde{b})\right]+\cdots \\
V \sim\left[v_{0}+\lambda E(-\gamma S+\alpha C)\right]+\hat{\beta}^{-1}\left[v_{1}+\lambda E\left\{C\left(\gamma g_{1}+\alpha f_{1}\right)+S\left(\alpha g_{1}-\gamma f_{1}\right)\right\}-E^{2} f_{1} / 2 \alpha\right]+ \\
\hat{\beta}^{-2}\left[v_{2}+\lambda E\left\{C\left(\gamma g_{2}+\alpha f_{2}\right)+S\left(\alpha g_{2}-\gamma f_{2}\right)\right\}+E^{2} \bar{c} / 2 \alpha+E^{3}(C \hat{a}+S \hat{b})\right]+\cdots
\end{gathered}
$$

where symbols appearing on the right-hand sides are given in Appendix.

Of interest are the longitudinal and transverse components of the shear stress at the surface, as well as the farfield speed. They are represented, respectively, by

$$
\begin{gathered}
F^{\prime}(0) \sim-\alpha+\hat{\beta}^{-1}\left(\alpha f_{1}+\gamma g_{1}\right)+\hat{\beta}^{-2}\left(-\alpha f_{2}+\gamma g_{2}-2 \alpha \bar{c}-3 \alpha \bar{a}+\gamma \bar{b}\right)+\cdots \\
G^{\prime}(0) \sim-\gamma+\hat{\beta}^{-1}\left(\alpha g_{1}-\gamma f_{1}\right)+\hat{\beta}^{-2}\left(-\alpha g_{2}-\gamma f_{2}-2 \alpha \tilde{c}-3 \alpha \tilde{a}+\gamma \tilde{b}\right)+\cdots \\
V(\infty) \sim v_{0}+\hat{\beta}^{-1} v_{1}+\hat{\beta}^{-2} v_{2}+\cdots=V_{\infty}
\end{gathered}
$$

The expansion for the pressure can be obtained from

$$
Q=-F+\frac{1}{2} \hat{\beta}^{-1}\left(V_{\infty}^{2}-V^{2}\right)
$$


which is the result of integrating Equation (4d) and the use of Equation (4a) and Condition (4j). This is not given here for brevity.

The above expansions describe how the flow behaves as $\beta \sim \infty$ with fixed $m$. They reveal a sinusoidal behavior that dies out exponentially as we move away from the surface. This behavior is solely due to the Hall effect, which is also responsible for the presence of the transverse velocity component $G$. When the Hall current is neglected; i.e. $m=0$, Expansion (6b) leads to $\gamma=0$, Expansion (6d) gives $G=0$, Expansions (6c) and (6e) reduce to $F=E$ and $V=V_{\mathrm{s}}+(E-1) / \alpha$, respectively, while Expansion (6a) translates to $\alpha$ satisfying $\alpha^{2}+\hat{\beta}^{-1}\left(V_{\mathrm{s}} \alpha-1\right)=1$. These results coincide with the exact solutions of the degenerate cases of Gupta and Gupta [2] and Andersson [3].

Expansions (6) describe the limiting case when $\beta \sim \infty$ with $m=\mathcal{O}\left(\beta^{1 / 2}\right)$, as well. All we need is to set $\gamma=\alpha$. It should, however, be noted that, now, as $\hat{\beta}=\mathcal{O}\left(\beta^{1 / 2}\right)$, the stretching (3) is weaker; being $\mathcal{O}\left(\beta^{1 / 4}\right)$. Moreover, the perturbations to the basic flow proceed in powers of $\beta^{-1 / 2}$.

\section{Results and Discussion}

The asymptotic expansions obtained above are tested against corresponding numerical results. The problem described by Equation (4) is solved numerically in double precision, using Keller's two-point, second-order-accurate, finite-difference scheme [11]. A uniform step size $\Delta \eta=0.01$ is used on a finite domain $0 \leq \eta \leq \eta_{\infty}$. The value of $\eta_{\infty}$ is chosen sufficiently large in order to insure the asymptotic satisfaction of the farfield conditions $(4 h, j)$. (As pointed out by Pantokratoras [12], a small value of $\eta_{\infty}$ can lead to erroneous results.) The non-linear terms are quasi-linearized, and an iterative procedure is implemented; terminating when the maximum errors in $F^{\prime}(0), G^{\prime}(0)$, and $H(\infty)$ become less than $10^{-10}$.

The numerical results exhibit the attenuating sinusoidal behavior predicted by the asymptotic analysis. This is clearly illustrated in Fig. 1 showing the longitudinal and transverse velocity profiles $F(\eta)$ and $G(\eta)$, when $\beta=20$, $m=10, V_{\mathrm{s}}=20$, and $\eta_{\infty}=500$. However, at such low value of $\beta$, a fixed period of oscillation is not sustained. When $\beta$ is increased to100 the $F$ and $G$ profiles, respectively, cross the zero line first at $\eta \approx 7.79$ and 15.44 then at $\eta \approx 23.15$ and 30.87; thus sustaining the same period $\tau \approx 30.8$. The two profiles cross the zero line several times later, but with much smaller magnitudes; maintaining the same period, though.

How quantitatively useful the asymptotic expansions of the previous section can be, is next investigated. To generate numerical values, for given $\beta, m$ and $V_{\mathrm{s}}$, we need to determine the coefficients $\alpha, \gamma, \lambda, v_{0}, f_{1}, g_{1}, v_{1} \ldots$, etc. To that end, we use Expansions (6a) and (6b) and the pertinent equations of Appendix A to obtain expansions of these coefficients in the form (5). In particular, the coefficients of the expansions which are involved to $\theta\left(\hat{\beta}^{-1}\right)$ are found to be $\alpha_{0}=\left[\frac{1}{2}\left\{\hat{n}+\left(\hat{m}^{2}+\hat{n}^{2}\right)^{1 / 2}\right\}\right]^{1 / 2}, \quad \gamma_{0}=\hat{m} / 2 \alpha_{0}, \quad \lambda_{0}=\left(\alpha_{0}^{2}+\gamma_{0}^{2}\right)^{-1}, \quad v_{00}=V_{\mathrm{s}}-\lambda_{0} \alpha_{0}, \quad \alpha_{1}=-\frac{1}{2} v_{00}$,

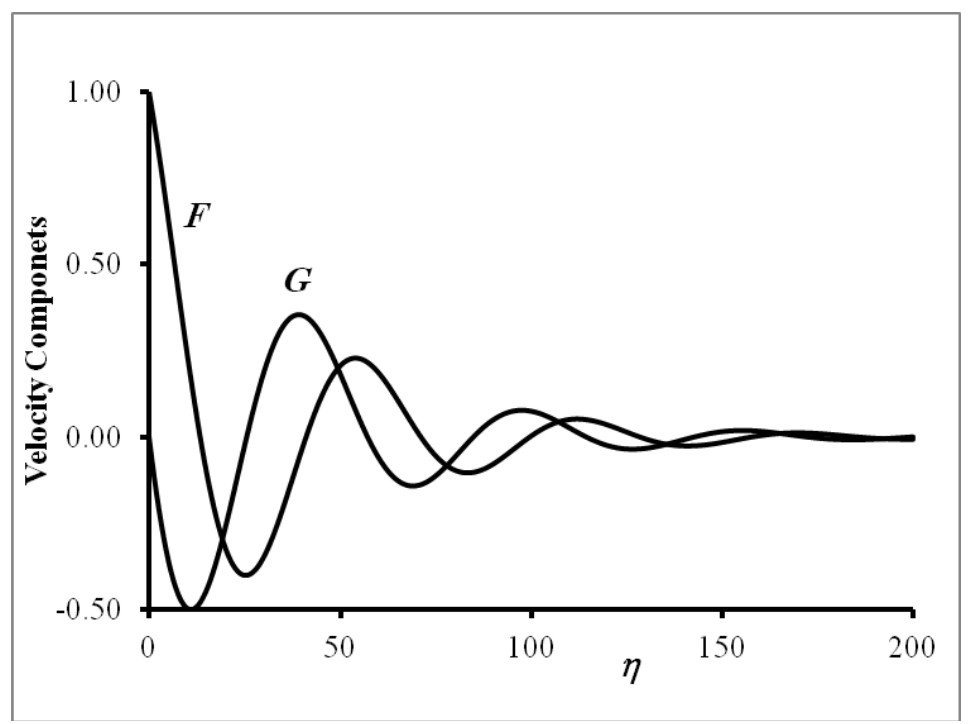

Figure 1. Velocity profiles; $\beta=20, m=10, V_{\mathrm{s}}=20$. 
$\gamma_{1}=0, \quad \lambda_{1}=-2 \lambda_{0}^{2}\left(\alpha_{0} \alpha_{1}+\gamma_{0} \gamma_{1}\right), \quad v_{01}=-\left(\lambda_{0} \alpha_{1}+\lambda_{1} \alpha_{0}\right), \quad f_{10}=-\lambda_{0} \gamma_{0}\left(\gamma_{0} \theta_{1}+\alpha_{0} \theta_{2}\right) / \theta_{3}, \quad g_{10}=\lambda_{0} \gamma_{0}\left(\alpha_{0} \theta_{1}-\gamma_{0} \theta_{2}\right) / \theta_{3}$, and $v_{10}=-\lambda_{0}\left(\hat{n} f_{10}+\hat{m} g_{10}\right) / 2 \alpha_{0}$; where $\theta_{1}=3 \alpha_{0}^{2}+\gamma_{0}^{2}, \theta_{2}=\hat{m}$, and $\theta_{3}=\theta_{1}^{2}+\theta_{2}^{2}$. The following expansions are also obtained

$$
\begin{gathered}
F^{\prime}(0)=-\alpha_{0}+\hat{\beta}^{-1}\left(-\alpha_{1}+\alpha_{0} f_{10}+\gamma_{0} g_{10}\right)+\Theta\left(\hat{\beta}^{-2}\right) \\
G^{\prime}(0)=-\gamma_{0}+\hat{\beta}^{-1}\left(-\gamma_{1}+\alpha_{0} g_{10}-\gamma_{0} f_{10}\right)+\Theta\left(\hat{\beta}^{-2}\right) \\
V(\infty)=v_{00}+\hat{\beta}^{-1}\left(v_{01}+v_{10}\right)+\mathcal{O}\left(\beta^{-2}\right)
\end{gathered}
$$

Results of the two-term asymptotic expansions (i.e., up to $\left.\Theta\left(\hat{\beta}^{-1}\right)\right)$ are proved to be in close agreement with the numerical results, even at values of $\beta$ as low as 20. This is illustrated in Figures 2 and $\mathbf{3}$ for $F^{\prime}(0)$ and $G^{\prime}(0)$ with different values of $m$, and in Figure 4 for $V(\infty)$ with different values of $V_{\mathrm{s}}$.

The period of the sinusoidal oscillations $\tau=2 \pi / \gamma$ takes the form

$$
\tau=4 \pi\left[\frac{1}{2}\left\{\hat{n}+\left(\hat{m}^{2}+\hat{n}^{2}\right)^{1 / 2}\right\}\right]^{1 / 2} / \hat{m}+\Theta\left(\hat{\beta}^{-2}\right)
$$

For $m=2$, 4, 6, 8, 10 Equation (9) gives $\tau=17.87,20.73$, 23.97, 26.96, 29.68, respectively, which are close to the corresponding numerically predicted values $\tau \approx 17.86,20.75,24.01,27.01,29.76$ obtained with $\beta=100$ and $V_{\mathrm{s}}=0$. That is, $\tau$ is independent of $V_{\mathrm{s}}$ to $\mathcal{O}\left(\hat{\beta}^{-2}\right)$, is also tested numerically. Varying $V_{\mathrm{s}}$ between -10 and 10 , with $\beta=100$ and $m=1$, gives $\tau=19.54 \pm 0.04$, as compared to the asymptotic value $\tau \approx 19.53$.

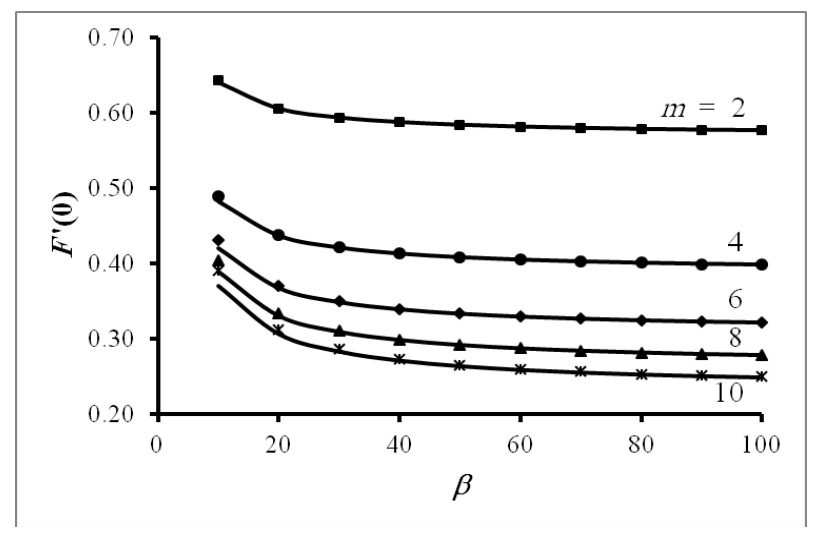

Figure 2. Longitudinal surface shear $F^{\prime}(0)$ vs. $\beta$, with different values of $m ; V_{\mathrm{s}}=0$. (Solid lines $\equiv$ numerical, markers $\equiv$ asymptotic).

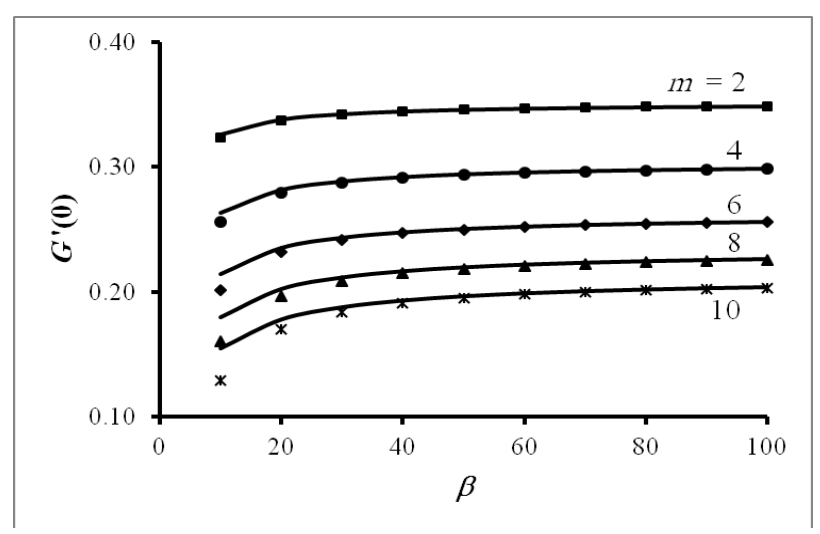

Figure 3. Transverse surface shear $G^{\prime}(0)$ vs. $\beta$, with different values of $m ; V_{\mathrm{s}}=0$. (Solid lines $\equiv$ numerical, markers $\equiv$ asymptotic). 


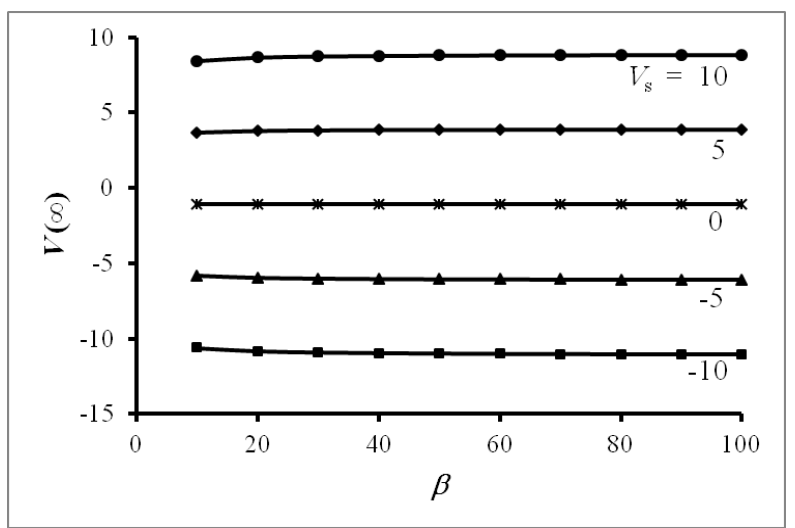

Figure 4. Farfield normal velocity $V(\infty)$ vs. $\beta$, with different values of $V_{\mathrm{s}} ; m=1$. (Solid lines $\equiv$ numerical, markers $\equiv$ asymptotic).

\section{Conclusion}

The limiting behavior of the MHD flow due to a porous stretching sheet has been studied, as the magnetic field grows indefinitely, taking into consideration the Hall current. Three-term uniformly-valid asymptotic expansions have been derived using parameter straining. The velocity components show sinusoidal behavior that attenuates exponentially, as we move away from the surface. (When the Hall current is neglected, the exponential decay becomes monotonic.) Finite difference solutions have also been calculated. The two-term asymptotic and the numerical solutions have shown excellent agreement both qualitatively and quantitatively.

\section{References}

[1] Crane, L.J. and Angew, Z. (1970) Flow Past a Stretching Plate. Zeitschrift für angewandte Mathematik und Physik ZAMP, 21, 645. http://dx.doi.org/10.1007/BF01587695

[2] Gupta, P.S. and Gupta, A.S. (1977) Heat and Mass Transfer on a Stretching Sheet with Suction or Blowing. Canadian Journal of Chemical Engineering, 55, 744. http://dx.doi.org/10.1002/cjce.5450550619

[3] Andersson, H.I. (1995) An Exact Solution of the Navier-Stokes Equations for Magnetohydrodynamic Flow. Acta Mechanica, 113, 241. http://dx.doi.org/10.1007/BF01212646

[4] Andersson, H.I. (2002) Slip Flow Past a Stretching Surface. Acta Mechanica, 158, 121. http://dx.doi.org/10.1007/BF01463174

[5] Wang, C.Y. (2009) Analysis of Viscous Flow Due to a Stretching Sheet with Surface Slip and Suction. Nonlinear Analysis: Real World Applications, 10, 375. http://dx.doi.org/10.1016/j.nonrwa.2007.09.013

[6] Abel, M.S. and Nandeppanavar, M.M. (2009) Heat Transfer in MHD Viscoelastic Boundary Layer Flow over a Stretching Sheet with Non-Uniform Heat Source/Sink. Communications in Nonlinear Science and Numerical Simulation, 14, 2120. http://dx.doi.org/10.1016/j.cnsns.2008.06.004

[7] Kumar, H. (2011) Heat Transfer over a Stretching Porous Sheet Subjected to Power Law Heat Flux in Presence of Heat Source. Thermal Science, 15, S187. http://dx.doi.org/10.2298/TSCI100331074K

[8] Vajravelu, K. and Rollins, D. (2004) Hydromagnetic Flow of a Second Grade Fluid over a Stretching Sheet. Applied Mathematics and Computation, 148, 783. http://dx.doi.org/10.1016/S0096-3003(02)00942-6

[9] Nayfeh, A.H. (1973) Perturbation Methods. John Wiley, New York.

[10] Sutton, G.W. and Sherman, A. (1965) Engineering Magnetohydrodynamics. McGraw-Hill, New York.

[11] Keller, H.B. (1969) Accurate Difference Methods for Linear Ordinary Differential Systems Subject to Linear Constraints. SIAM Journal on Numerical Analysis, 6, 8. http://dx.doi.org/10.1137/0706002

[12] Pantokratoras, A. (2009) A Common Error Made in Investigation of Boundary Layer Flows. Applied Mathematical Modelling, 33, 413. http://dx.doi.org/10.1016/j.apm.2007.11.009 


\section{Appendix}

Given below are the expressions and relations that define the different symbols appearing in Expansions (6). For conciseness, let

$$
E=e^{-a \eta}, C=\cos \gamma \eta, S=\sin \gamma \eta, \lambda=\left(a^{2}+\gamma^{2}\right)^{-1}
$$

then

$$
v_{0}=V_{\mathrm{s}}-\lambda \alpha
$$

$f_{1}, g_{1}$ and $v_{1}$ are obtained from

$$
\begin{gathered}
\left(3 \alpha^{2}+\gamma^{2}\right) f_{1}+2 \alpha \gamma g_{1}=-\lambda \gamma^{2} \\
-2 \alpha \gamma f_{1}+\left(3 \alpha^{2}+\gamma^{2}\right) g_{1}=\lambda \alpha \gamma \\
v_{1}=-\lambda\left(\gamma g_{1}+\alpha f_{1}\right)+f_{1} / 2 \alpha
\end{gathered}
$$

$\bar{C}$ and $\tilde{c}$ satisfy

$$
\begin{aligned}
& \left(3 \alpha^{2}+\gamma^{2}\right) \bar{c}+2 \alpha \gamma \tilde{c}=v_{0}\left(\gamma g_{1}+\alpha f_{1}\right)+2 \lambda \gamma^{2} f_{1} \\
& -2 \alpha \gamma \bar{c}+\left(3 \alpha^{2}+\gamma^{2}\right) \tilde{c}=v_{0}\left(\alpha g_{1}-\gamma f_{1}\right)-2 \lambda \alpha \gamma f_{1}
\end{aligned}
$$

$\bar{a}, \bar{b}, \tilde{a}$, and $\tilde{b}$ satisfy

$$
\begin{gathered}
8 \alpha^{2} \bar{a}-6 \alpha \gamma \bar{b}+2 \alpha \gamma \tilde{a}=\lambda\left(\alpha^{2}-3 \gamma^{2}\right) f_{1} / 2 \\
6 \alpha \gamma \bar{a}+8 \alpha^{2} \bar{b}+2 \alpha \gamma \tilde{b}=\left(\gamma^{2}-3 \alpha^{2}\right) \lambda f_{1} \gamma / 2 \alpha \\
-2 \alpha \gamma \bar{a}+8 \alpha^{2} \tilde{a}-6 \alpha \gamma \tilde{b}=\left(\alpha^{2}-\gamma^{2}\right) \lambda g_{1}+\gamma f_{1} / 2 \alpha \\
-2 \alpha \gamma \bar{b}+6 \alpha \gamma \tilde{a}+8 \alpha^{2} \tilde{b}=f_{1} / 2-2 \lambda \alpha \gamma g_{1}
\end{gathered}
$$

$\hat{a}$ and $\hat{b}$ satisfy

$$
\begin{array}{r}
3 \alpha \hat{a}-\gamma \hat{b}=\bar{a} \\
\gamma \hat{a}+3 \alpha \hat{b}=\bar{b}
\end{array}
$$

and finally $f_{2}, g_{2}$, and $v_{2}$ are obtained from

$$
\begin{gathered}
f_{2}=-\bar{c}-\bar{a} \\
g_{2}=-\tilde{c}-\tilde{a} \\
v_{2}=-\lambda\left(\gamma g_{2}+\alpha f_{2}\right)-\bar{c} / 2 \alpha-\hat{a}
\end{gathered}
$$

